\title{
Gerenciamento de resíduos sólidos de odontologia em postos de saúde da rede municipal de Belo Horizonte, Brasil
}

\author{
Michel William Nazar, ${ }^{1}$ Isabela Almeida Pordeus ${ }^{2}$ \\ e Marcos Azeredo Furquim Werneck ${ }^{2}$
}

Como citar Nazar MW, Pordeus IA, Werneck MAF. Gerenciamento de resíduos sólidos de odontologia em postos de saúde da rede municipal de Belo Horizonte, Brasil. Rev Panam Salud Publica. 2005;17(4):237-42.

RESUMO Objetivo. Verificar a conformidade do processo de gerenciamento dos resíduos sólidos odontológicos pelas unidades básicas de saúde da rede municipal de Belo Horizonte frente à legislação vigente.

Método. Foram avaliadas 54 unidades básicas de saúde das 105 unidades da rede municipal que prestavam atendimento odontológico. Em cada unidade, foram entrevistados o gerente, um atendente de consultório dentário e um auxiliar de serviços gerais. Com base nos requisitos estabelecidos pelo Manual de Gerenciamento de Resíduos de Serviços de Saúde de Belo Horizonte, foram avaliados os seguinte aspectos: técnicos e operacionais (classificação e caracterização dos resíduos, processos de minimização, segregação, tratamento prévio, acondicionamento, coleta e transporte internos e externos e abrigo externo); gerais e organizacionais (fiscalização, área construída, licenciamento ambiental, planta baixa com discriminação das áreas geradoras de resíduos e presença de responsável técnico); recursos humanos (registro e controle de vacinação dos trabalhadores, programa de segurança e medicina do trabalho, programa de prevenção de riscos ambientais, programa de controle médico e saúde ocupacional, comissão de controle de infecção hospitalar e treinamento em manejo de resíduos de serviços de saúde).

Resultados. Cada unidade básica de saúde gerava uma média de 270 litros de resíduos por dia. Nenhuma possuía um plano de gerenciamento de resíduos de serviços de saúde. Os únicos itens em conformidade com a legislação em todas as unidades foram: as agulhas e o mercúrio eram segregados; eram utilizados recipientes de papelão resistente com simbologia adequada para acondicionamento dos resíduos perfurocortantes; e a coleta e transporte internos de resíduos eram feitos diariamente.

Conclusão. Enquanto não forem estabelecidos os riscos reais de cada classe de resíduos, todas deveriam ser consideradas como potencialmente perigosas. Por outro lado, uma lei em vigor por si só não garante que a saúde da população está protegida. Antes de impor exigências legais, é preciso verificar até que ponto os órgãos públicos estão preparados para cumpri-las. A realização de pesquisas sobre gerenciamento de resíduos é importante para fundamentar a legislação.

Palavras-chave Gerenciamento de resíduos, legislação sanitária, resíduos odontológicos, Brasil.

1 Universidade Federal de Minas Gerais (UFMG), Faculdade de Odontologia, Programa de PósGraduação em Saúde Coletiva. Enviar correspondência para este autor no seguinte endereço: Rua Cordisburgo 12, apto. 301, Santa Inês, CEP
31080-060, Belo Horizonte, MG, Brasil. E-mail: michelnazar@ig.com.br

2 UFMG, Faculdade de Odontologia, Belo Horizonte (MG), Brasil.
O gerenciamento de resíduos deve ser implantado e implementado em qualquer estabelecimento que preste serviços de atenção à saúde, conforme 
determinam as legislações federal, estadual e municipal no Brasil $(1,2)$. Em Belo Horizonte, capital do Estado de Minas Gerais, com aproximadamente 2 milhões de habitantes, a legislação municipal (2) impõe a implantação de um plano de gerenciamento de resíduos de serviços de saúde (PGRSS) em todos os estabelecimentos desse tipo. $\mathrm{O}$ PGRSS tem como referência principal o manual produzido pela Comissão Permanente de Apoio ao Gerenciamento de Resíduos de Serviços de Saúde (COPAGRESS) de Belo Horizonte (3), que é vinculada à Secretaria Municipal de Limpeza Urbana. O manual, em conformidade com o que determina o Conselho Nacional de Meio Ambiente (CONAMA) (1), estabelece que o PGRSS deve abranger aspectos técnicos e operacionais, aspectos gerais e organizacionais e aspectos de recursos humanos. O foco do manual é orientar a implantação, a implementação e o acompanhamento de uma política de gerenciamento de resíduos de saúde.

Vários autores em todo o mundo relatam que os riscos para a saúde pública gerados pelos resíduos de serviços de saúde são comparáveis aos riscos ocasionados pelos resíduos domésticos, o que dispensaria, para os primeiros, uma atenção especial no que diz respeito ao tratamento, à coleta e à disposição final (4-8). A exceção unânime são os resíduos perfurocortantes e as culturas microbiológicas. $\mathrm{O}$ risco de contaminação pelo manuseio desses resíduos é alto, tanto no momento da geração, do acondicionamento e do descarte, quanto durante a coleta externa e a disposição final, devido às suas características físicas e ao seu potencial de contaminação através de microorganismos retidos, requerendo normas seguras de manuseio e acondicionamento (4-12).

Conforme a classificação do CONAMA (1), os resíduos gerados pelas práticas odontológicas se enquadram nos grupos A (infectantes ou biológicos), B (químicos) e D (comuns). No grupo $\mathrm{A}$, exigem atenção especial os resíduos perfurocortantes. No grupo B merece atenção o mercúrio metálico, que também exige critérios especiais de manuseio, acondicio- namento e destinação final. $\mathrm{O}$ maior risco para a equipe odontológica em relação ao mercúrio está no momento da preparação do amálgama, quando ocorre o aquecimento e a conseqüente liberação do vapor de mercúrio (13). Para a população, o risco está na organificação e biomagnificação do mercúrio lançado no ambiente natural (14).

Dessa forma, o objetivo do presente estudo foi verificar a conformidade do processo de gerenciamento dos resíduos sólidos odontológicos nas unidades básicas de saúde da rede municipal de Belo Horizonte em termos das orientações contidas no Manual de Gerenciamento de Resíduos de Serviços de Saúde de Belo Horizonte.

\section{MATERIAIS E MÉTODOS}

Foi adotada a metodologia quantitativa, utilizando-se a técnica de entrevista estruturada, através de formulário, conduzida por um único pesquisador (MWN) (15). Foram realizados um pré-teste e um estudo piloto antes do estudo principal. A amostra foi calculada em 51 unidades básicas de saúde $(16,17)$, corrigida para uma população finita (18) de 105 unidades que ofertavam serviços de odontologia. A essas 51 unidades foram acrescidos $10 \%$, que correspondiam à margem de erro, para um total de 56 unidades. A amostra principal foi estratificada (19) pelos nove distritos sanitários que compõem a rede municipal de saúde de Belo Horizonte e randomizada. Em cada unidade básica de saúde visitada, foram entrevistados o gerente, um atendente de consultório dentário e um auxiliar de serviços gerais. Foram visitadas 64 unidades, sendo cinco no pré-teste e cinco no estudo piloto, para adequação do instrumento metodológico de pesquisa, e 54 no estudo principal. Duas das 56 unidades selecionadas não foram avaliadas porque seus gerentes não estavam disponíveis durante o período do trabalho de campo (julho a setembro de 2001).

Foram avaliadas todas as etapas dos três aspectos determinados pela legislação vigente (3). Dos aspectos técnicos e operacionais foram avaliados: classifi- cação e caracterização dos resíduos, minimização (redução, reutilização, recuperação, reciclagem ou substituição de um determinado resíduo por outro menos perigoso), segregação (separação dos resíduos no momento de sua geração segundo suas características físicas, químicas, biológicas e radiológicas), tratamento prévio, acondicionamento, coleta e transporte internos e externos e existência de abrigo externo. Dos aspectos gerais e organizacionais foram avaliados: fiscalização, área construída, licenciamento ambiental, planta baixa com discriminação das áreas geradoras de resíduos e presença de responsável técnico. Dos aspectos de recursos humanos foram avaliados: registro e controle de vacinação dos trabalhadores, programa de segurança e medicina do trabalho, programa de prevenção de riscos ambientais, programa de controle médico e saúde ocupacional, comissão de controle de infecção hospitalar e treinamento em manejo de resíduos de serviços de saúde.

Para cada uma das três classe de profissionais a serem entrevistados, foi elaborado um formulário com perguntas fechadas. Esses formulários foram corrigidos e aperfeiçoados no pré-teste e no estudo piloto. Foi utilizado, também, um formulário de observação, preenchido pelo entrevistador durante as visitas às unidades pesquisadas, com a intenção de validar ou corrigir as respostas dos entrevistados.

Por ser este o primeiro estudo baseado na legislação municipal, abordando todas as variáveis determinadas nessa legislação, e pela dificuldade em encontrar estudos publicados que permitissem uma análise estatística comparativa mais aprofundada de todas as variáveis apresentadas, optou-se por analisar os resultados através de freqüência relativa simples.

\section{RESULTADOS}

\section{Caracterização dos entrevistados}

Dos 54 gerentes entrevistados, 50 $(92,6 \%)$ eram mulheres. Todos tinham curso superior completo (mínimo de 15 anos de estudo). Vinte $(37,0 \%)$ exer- 
ciam a função há menos de 5 anos, e 21 $(38,95 \%)$ tinham entre 5 e 10 anos no exercício da função. Todos os atendentes de consultório dentário eram mulheres; $37(68,5 \%)$ tinham entre 5 e 10 anos no exercício da função e 38 $(70,4 \%)$ tinham completado o ensino secundário (11 anos de estudo). Entre os auxiliares de serviços gerais, 51 $(94,4 \%)$ eram mulheres. Vinte e sete (50\%) tinham menos de 5 anos no exercício da função e $38(70,4 \%)$ não tinham o ensino fundamental completo (menos de 8 anos de estudo). Os gerentes e atendentes faziam parte do quadro permanente de servidores da prefeitura. Entretanto, 48 auxiliares $(88,9 \%)$ eram contratados de empresas prestadoras de serviços terceirizados de conservação e limpeza.

\section{Aspectos técnicos e operacionais}

Neste estudo, estimou-se que o volume médio de resíduos gerados por unidade básica de saúde era de 270 litros por dia. Nenhuma das unidades possuía um plano de gerenciamento de resíduos de serviços de saúde.

Das 54 unidades analisadas, 98,1\% não tinham os seus resíduos caracterizados. Nenhuma das unidades pesquisadas adotava qualquer processo de minimização. Somente as agulhas e o mercúrio eram segregados (100\%). Entretanto, não eram separados dos resíduos comuns os outros resíduos infectantes gerados, tais como elementos dentários extraídos; tecidos moles de pequenas cirurgias; algodões ou gazes com exsudatos corporais, saliva ou sangue; e outros resíduos perfurocortantes, como limas endodônticas, brocas, matrizes de aço para amálgama, fios ortodônticos e, em alguns casos, lâminas de bisturi. Da mesma forma, 98,1\% das unidades não faziam tratamento prévio de seus resíduos.

Todas as unidades pesquisadas utilizavam sacos de plástico brancos, sem o símbolo de lixo infectante, para o acondicionamento tanto dos resíduos infectantes como dos resíduos comuns, o que é inadequado para o acondicionamento de ambos os tipos de resíduos. Esse fato gera risco para os indivíduos envolvidos nas etapas de gerenciamento interno e externo dos resíduos, assim como para a população em geral. Por outro lado, o recipiente de papelão resistente com simbologia adequada para acondicionamento dos resíduos perfurocortantes foi encontrado em todas as unidades pesquisadas. Em 25 (46,3\%) unidades, os resíduos mercuriais eram armazenados em recipientes de vidro com água e, em 29 (53,7\%), em recipientes plásticos com água. Nenhuma unidade encaminhava os resíduos de mercúrio para a reciclagem, conforme recomenda o manual (3).

A coleta e o transporte internos de resíduos foram considerados adequados em todas as unidades pesquisadas no que diz respeito à freqüência, no mínimo diária. Quanto à rota de transporte dentro da unidade de saúde, apenas seis $(11,1 \%)$ unidades foram consideradas adequadas. Em $48(88,9 \%)$, a rota da coleta interna coincidia com a rota dos funcionários e usuários dentro da unidade de saúde, expondo-os a riscos indesejáveis frente à possibilidade de acidentes. Somente $27(50 \%)$ das unidades pesquisadas possuíam algum tipo de abrigo externo para resíduos. Entretanto, nenhum deles foi considerado adequado.

Segundo o manual da COPAGRESS (3), a coleta externa dos resíduos infectantes ou biológicos deve ser feita diariamente ou, no máximo, em 48 horas, quando há abrigo externo adequado. Ainda, os resíduos comuns devem ser coletados pelo serviço domiciliar de coleta, e os resíduos especiais, pela coleta especial. Porém, em 34 (62,2\%) unidades, a coleta especial recolhia indiscriminadamente todos os resíduos, infectantes e comuns. Em $15 \quad(27,8 \%)$ unidades, a coleta especial recolhia somente os resíduos perfurocortantes e, em cinco $(9,3 \%)$, a coleta domiciliar recolhia todos os resíduos, infectantes e comuns. Somente em duas $(3,7 \%)$ unidades havia coleta externa diariamente.

\section{Aspectos gerais e organizacionais}

Segundo o manual da COPAGRESS (3), é obrigatória a fiscalização regular das unidades de saúde por órgãos competentes, como a secretaria municipal de limpeza urbana ou as secretarias de meio ambiente e vigilância sanitária. A fiscalização pode ter ação punitiva, mas pode ter, também, ações educativas, estimuladoras e incentivadoras. Apenas 10 (18,6\%) das unidades pesquisadas haviam sido fiscalizadas por órgãos competentes.

Das unidades pesquisadas, 40 $(74,1 \%)$ tinham mais de $80 \mathrm{~m}^{2}$ de área construída e, portanto, deveriam ter uma sala de resíduos para acondicionamento intermediário. Não foi encontrada a sala de resíduos em nenhuma das unidades pesquisadas. Em nenhuma das unidades pesquisadas foi apresentada a licença ambiental ou a planta baixa com discriminação das áreas geradoras de resíduos, conforme exige a legislação (3). A planta baixa possibilita uma visão geral da unidade de saúde, permitindo a identificação dos pontos de geração de cada tipo de resíduo e um melhor planejamento do fluxo de coleta e transporte desses resíduos. Além disso, nenhuma unidade possuía um responsável técnico para o gerenciamento dos resíduos de serviços de saúde.

\section{Aspectos de recursos humanos}

Entre as unidades pesquisadas, nenhuma conhecia a existência, seja na unidade, seja em instâncias superiores da prefeitura, dos programas de prevenção de riscos ambientais (PPRA) e de controle médico e saúde ocupacional (PCMSO). Somente o programa de saúde e medicina do trabalho (PSMT), cujo objetivo é a informação e a prevenção dos acidentes de trabalho, era conhecido pela maioria $(92,6 \%)$. Entretanto, por estar ligado a instâncias superiores da prefeitura, o PSMT só era acionado, conforme relataram os gerentes, se houvesse necessidade (provavelmente após a ocorrência de um acidente). Também não havia, em nenhuma das unidades pesquisadas, uma comissão de controle de infecção hospitalar (CCIH), e somente $12(22,2 \%)$ unidades apresentaram o registro de vacinação de seus funcionários. 
Três gerentes $(5,6 \%)$, oito atendentes $(14,8 \%)$ e 12 auxiliares $(22,2 \%)$ relataram ter freqüentado um curso sobre manejo de resíduos de saúde no último ano. Relataram nunca ter tido um curso sobre manejo de resíduos, enquanto no exercício da função em que atuavam, 47 $(86,9 \%)$ gerentes, $46(85,2 \%)$ atendentes e $31(57,4 \%)$ auxiliares.

\section{DISCUSSÃO}

Na prática, o gerenciamento de resíduos odontológicos nas unidades básicas da rede municipal de saúde de Belo Horizonte estava muito aquém das exigências impostas e idealizadas pela legislação municipal vigente. Na maioria das unidades, os resíduos nem sequer eram classificados ou caracterizados, ou seja, quantificados, apesar de serem esses passos fundamentais para o gerenciamento de resíduos $(7,8,20)$.

Além disso, nenhuma das unidades pesquisadas adotava qualquer tipo de processo de minimização, sendo esta uma etapa básica do GRSS $(3,21)$. Para o mercúrio, a reciclagem, a diminuição do uso ou a substituição do material são os processos de minimização mais recomendados. Além disso, nenhum dos resíduos infectantes gerados, com exceção das agulhas e do mercúrio, eram segregados dos resíduos comuns. Na Nova Zelândia, Treasure e Treasure (22) encontraram um índice de $80,3 \%$ de segregação de perfurocortantes e de $83,7 \%$ para o mercúrio. $\mathrm{Na}$ Austrália, Cannata et al. (23) constataram que $100 \%$ dos resíduos infectantes odontológicos eram segregados dos resíduos comuns. Para a Organização Mundial da Saúde (OMS), os principais resíduos gerados em consultórios odontológicos são resíduos infectantes, perfurocortantes e resíduos contendo grande conteúdo de metal pesado, como o amálgama odontológico (10). Enquanto não forem estabelecidos, por pesquisas científicas, os riscos reais de cada classe de resíduos, todas as classes e subclasses de resíduos odontológicos deveriam ser segregadas. A segregação dos resíduos na fonte permite a identificação, a classificação, a caracterização, a escolha de processos de minimização, o tratamento prévio, o acondicionamento, $\mathrm{o}$ armazenamento, a coleta e o transporte e a disposição final correta, adequada e segura para cada tipo de resíduo. A segregação é importante, ainda, para diminuir o volume dos resíduos infectantes, já que, quando os resíduos infectantes são misturados aos resíduos comuns, estes passam também a ser considerados infectantes. Sem a segregação adequada dos resíduos, todas as outras etapas do gerenciamento ficam comprometidas.

Neste estudo, 98,1\% das unidades não faziam tratamento prévio de seus resíduos. Outro estudo feito no Brasil (24), na Cidade de João Pessoa, capital do Estado da Paraíba, observou que $67,86 \%$ das clínicas odontológicas não realizavam nenhum tipo de tratamento prévio dos resíduos, e que $5,35 \%$ utilizavam processos químicos na sua realização. Morel e Bertussi Filho (25) alertam para a necessidade de cuidado com o uso de substâncias químicas no tratamento prévio, pois algumas podem gerar resíduos perigosos à saúde pública e ao ambiente.

No Estado da Paraíba, Nobrega et al. (24) relataram que 40,7\% dos hospitais e centros de saúde investigados acondicionavam seus resíduos, e desses, 29,6\% utilizavam sacos adequados. Em Belo Horizonte, observamos que, embora fossem utilizados sacos brancos, esses não possuíam o símbolo de lixo infectante, e eram usados para acondicionar tanto os resíduos infectantes como os resíduos comuns. Essa situação pode gerar dúvidas sobre o conteúdo de cada volume e levar à desqualificação ou banalização da simbologia e da cor branca como referência dos serviços de saúde. $O$ fato de que todas as unidades pesquisadas usavam o recipiente de papelão resistente com simbologia adequada para acondicionamento dos resíduos perfurocortantes pode ter advindo da conscientização dos profissionais de odontologia sobre o alto grau de risco representado por esses resíduos frente à possibilidade de acidentes que levem à contaminação com os vírus da hepatite B e da AIDS. As entidades representativas da classe odontológica no
Brasil têm dado ênfase aos cuidados com os instrumentos perfurocortantes, promovendo cursos e treinamentos constantes sobre o tema junto à comunidade odontológica. Entre os autores pesquisados, houve unanimidade sobre a necessidade incondicional de um gerenciamento correto e rigoroso para esses resíduos. Os resíduos perfurocortantes eram adequadamente acondicionados em $73,3 \%$ dos locais pesquisados na Nova Zelândia (22) e em 85,7\% dos locais estudados por Nobrega et al. (24) no Brasil.

A glicerina é mais indicada do que a água para armazenar os resíduos mercuriais (26). Os recipientes de plástico também são mais aconselháveis do que os de vidro, que quebram mais facilmente. $\mathrm{O}$ processo de minimização mais adequado para os resíduos de mercúrio e amalgama odontológicos é a reciclagem. A substituição do amálgama por algum material restaurador sem substâncias tóxicas, que se iguale ou supere as características técnicas e de manuseio, com custo igual ou inferior ao do amálgama, seria uma outra opção de minimização para este tipo de resíduo odontológico. No presente estudo, as unidades utilizavam recipientes de vidro com água $(46,3 \%)$ ou plástico com água $(53,7 \%)$ para armazenar os resíduos de mercúrio. Treasure e Treasure (22) relatam que em $62,6 \%$ dos locais pesquisados na Nova Zelândia, o amálgama era armazenado submerso em água, e em 21,1\%, era submerso em fixador de radiografias. Esses autores relataram ainda que $70,5 \%$ dos locais encaminhavam os resíduos mercuriais para a reciclagem. Também no Estado de Minas Gerais, Rink et al. (27) encontraram resíduos de mercúrio $(44,9 \%)$ e resíduos de amálgama (26,9\%) acondicionados em vidros com água, com o restante sendo disposto no ambiente através da pia, da cuspideira ou do lixo.

Os resultados acerca da rota de transporte interno dos resíduos, considerada inadequada em $89 \%$ das unidades, demonstram a falta de preocupação com o gerenciamento de resíduos no planejamento da construção ou locação das unidades de saúde pesquisadas. Quanto à coleta 
externa, considerada inadequada em todas as unidades visitadas, isso pode ser resultado do acondicionamento dos resíduos, que não permitia diferenciação entre os resíduos infectantes e os comuns. Dessa forma, era difícil para os trabalhadores da coleta externa especial identificar qual volume correspondia aos resíduos infectantes, a menos que se abrissem todos os sacos de lixo. Supomos que a opção dos trabalhadores da coleta especial, frente ao alto risco ao qual estariam expostos se abrissem os sacos, foi a de recolher todos os resíduos acondicionados em sacos brancos, ou a de recolher apenas os volumes com simbologia de identificação correta, no caso as caixas de perfurocortantes. Para a coleta domiciliar, a mesma situação poderia ser inferida.

Nenhuma das unidades em Belo Horizonte possuía plano de gerenciamento de resíduos de serviços de saúde, contra $25,9 \%$ das unidades públicas de saúde em João Pessoa (24). Para que um PGRSS seja implantado e funcione efetivamente, é necessário que a unidade de saúde esteja equipada adequadamente e que seus funcionários sejam estimulados, treinados e capacitados para o manejo adequado dos resíduos. Além disso, é necessário que os órgãos públicos de limpeza urbana, fiscalização, meio ambiente e educação trabalhem em conjunto, buscando a proteção da população, dos trabalhadores e do ambiente.

Quanto aos aspectos organizacionais, as unidades pesquisadas também ficavam muito aquém das exigências legais, não apresentando, por exemplo, uma sala de resíduos para acondicionamento intermediário, a licença ambiental ou a planta baixa com discriminação das áreas geradoras de resíduos. As unidades tampouco possuíam um responsável técnico para o gerenciamento dos resíduos de ser-

. Brasil, Ministério do Meio Ambiente, Conselho Nacional do Meio Ambiente. Resolução no 5 . Diário Oficial da União 31 de agosto de 1993: seção 1.

2. Belo Horizonte, Prefeitura Municipal, Secretaria Municipal de Governo. Decreto $\mathrm{n}^{\mathrm{o}}$ 10296, de 13 de julho de 2000. Diário Oficial viços de saúde, conforme exigido por lei. A presença de um profissional especializado na unidade de saúde é fundamental para o bom andamento do processo de gerenciamento de resíduos, facilitando o cumprimento de outras exigências legais (25). Também preocupa a falta de treinamento dos recursos humanos constatada neste trabalho, e o desconhecimento acerca de programas preventivos.

Não foram encontradas nas unidades as comissões de controle de infecção hospitalar. Para Guimarães Jr. (11), essas comissões têm por objetivo a vigilância epidemiológica, a educação na prevenção de riscos, a orientação sobre normas e condutas de biossegurança, a identificação de fatores de risco de infecção hospitalar, a definição das estratégias e prioridades das ações de biossegurança, além de liderar e apoiar o processo educacional contínuo $(25,28)$ em controle de infecção e biossegurança.

Os programas preventivos, como o de prevenção de riscos ambientais e de controle médico e saúde ocupacional, objetivam manter a integridade física dos trabalhadores através da avaliação dos riscos e das possibilidades de agravo à saúde no ambiente de trabalho. $\mathrm{O}$ manejo de resíduos exige o uso de tecnologia, que, por sua vez, exige treinamento e controle constantes $(3,29)$. Sem os programas citados, e sem a presença de uma comissão de controle de infecção, os trabalhadores envolvidos com o manejo de resíduos estavam expostos a um alto grau de risco e à mercê de sua própria sorte. Em relação a isso, observou-se que há um interesse maior das empresas terceirizadas do que da prefeitura em treinar seus funcionários, apesar de estarem muito aquém do recomendado. Isso contraria as inferências de $\mathrm{Fe}-$ rreira (7) sobre o menor interesse das empresas terceirizadas em treinar e capacitar seus funcionários devido à alta rotatividade desses trabalhadores.

\section{CONSIDERAÇÕES FINAIS}

A legislação deve, sempre, estar preocupada com o bem comum e a qualidade de vida das populações. Enquanto não forem estabelecidos os riscos reais de cada classe de resíduos, todas as classes e subclasses de resíduos odontológicos deveriam ser consideradas como potencialmente perigosas.

Por outro lado, a legislação deve ter como base as pesquisas científicas. A mera existência de uma lei não é suficiente para atingir o objetivo que se propõe, como no caso da legislação que rege a elaboração e implantação do PGRSS no Município de Belo Horizonte. Para tanto, o diálogo entre a ciência, os órgãos competentes, os trabalhadores e a população em geral é de fundamental importância na busca de soluções para os desafios que se apresentam frente a um ideal. Enquanto isso não ocorrer, a saúde e a qualidade de vida da população encontram-se vulneráveis aos riscos provenientes de um gerenciamento de resíduos de serviços de saúde ineficiente e inadequado. A prefeitura municipal de Belo Horizonte, ao exigir a implantação de um PGRSS altamente complexo, sem verificar se estava, ela mesma, preparada para cumpri-lo, corre o risco de ter a sua idoneidade legislativa questionada por aqueles que se encontram envolvidos com o gerenciamento desses resíduos especiais, como a população e os trabalhadores que atuam nessa área. A realização de pesquisas que abordem os mais variados aspectos referentes ao gerenciamento de resíduos é cada vez mais importante como forma de fundamentar a legislação.

\section{REFERÊNCIAS}

do Município 14 de julho de 2000:1-4 (vol. 6, $\mathrm{n}^{\circ}$ 1172).

3. Comissão Permanente de Apoio ao Gerenciamento dos Resíduos de Serviços de Saúde. Manual de gerenciamento de resíduos de serviços de saúde de Belo Horizonte, MG. Belo Horizonte: COPAGRESS; 1999.
4. Zanon U. Riscos infecciosos imputados ao lixo hospitalar. Realidade epidemiológica ou ficção sanitária? Rev Soc Bras Med Trop. 1990; 23(3):163-70.

5. Dugan SFX. Regulated medical waste: is any of it infectious? N Y State J Med. 1992;92(8): 349-52. 
6. Rutala WA, Mayhall CG. Medical waste. Infect Control Hosp Epidemiol. 1992;13(1):38-48.

7. Ferreira JA. Lixo hospitalar e domiciliar: semelhanças e diferenças. Estudo de caso no município do Rio de Janeiro [tese de doutorado]. Rio de Janeiro: Fundação Osvaldo Cruz, Escola Nacional de Saúde Pública; 1997.

8. Andrade JBL. Determinação da composição gravimétrica dos resíduos de serviços de saúde de diferentes tipos de estabelecimentos geradores. Em: Anais do $20^{\circ}$ Congresso Brasileiro de Engenharia Sanitária e Ambiental. Rio de Janeiro: Associação Brasileira de Engenharia Sanitária e Ambiental; 1999. Pp. 1666-72.

9. Nogueira JM. Lixo hospitalar. Em: Couto RC, Pedrosa TMG, Nogueira JM. Infecção hospitalar: epidemiologia, controle, gestão para a qualidade. 2aㅡ. ed. Rio de Janeiro: Medsi; 1999. Pp. 219-38.

10. Pruss A, Giroult E, Rushbrook P. Safe management of wastes from health-care activities. Genebra: World Health Organization; 1999.

11. Guimarães Jr J. Biossegurança e controle de infecção cruzada em consultórios odontológicos. São Paulo: Santos; 2001.

12. Salkin IF, Kennedy ME. Review of health impacts from microbiological hazards in healthcare wastes. Genebra: World Health Organization; 2001

13. Couto Jr MP. Mercúrio (Hg): contaminação só quando há negligência. Rev CROMG. 1996; 2(2):108-10.

14. Cota PL. Redução da contaminação mercurial em áreas de garimpo de ouro: desenvolvimento de uma alternativa tecnológica [disser- tação de mestrado]. Belo Horizonte: Escola de Engenharia, Universidade Federal de Minas Gerais; 1997.

15. Lakatos EM, Marconi MA. Fundamentos de metodologia científica. São Paulo: Atlas; 1991.

16. Luanga SK, Lemeshow S. Sample size determination in health studies: a practical manual. Genebra: World Health Organization; 1991.

17. Kirkwood BR. Essentials of medical statistics. Oxford: Blackwell Science; 1996.

18. Levy PS, Lemeshow S. Sampling of populations: methods and applications. New York: John Wiley \& Sons; 1991.

19. Pereira MG. Epidemiologia: teoria e prática. Rio de Janeiro: Guanabara Koogan; 1995.

20. Bertussi Filho LA. Resíduos de serviços de saúde: gerenciamento, tratamento e destinação final. Curitiba: Associação Brasileira de Engenharia Sanitária e Ambiental; 1994.

21. Rocca ACC, Lacovone AMMB, Barrotti AJ. Resíduos sólidos industriais. 2ª ed. São Paulo: Companhia de Tecnologia e Saneamento Básico; 1993

22. Treasure ET, Treasure P. An investigation of the disposal of hazardous wastes from New Zealand dental practices. Community Dent Oral Epidemiol. 1997;25(4):328-31.

23. Cannata S, Bek M, Baker P, Fett M. Infection control and contaminated waste disposal practices in Southern Sydney Area Health Service Dental Clinics. Aust Dent J. 1997;42(3): 199-202.

24. Nobrega CC, Paes RFC, Flores Neto JP, Lima JD, Ruberg C. Resíduos sólidos de serviços de saúde oriundos de clínicas odontológicas e clínicas veterinárias da cidade de João
Pessoa/PB-Brasil: resultados preliminares. Em: Anais do $27^{\circ}$ Congresso Interamericano de Engenharia Sanitária e Ambiental. Porto Alegre: Associação Brasileira de Engenharia Sanitária e Ambiental; 2000.

25. Morel MMO, Bertussi Filho LA. Resíduos de serviços de saúde. Em: Rodrigues EAC, Mendonça JS, Amarante JMB, Grinbaum RS, Richtmann R. Infecções hospitalares: prevenção e controle. São Paulo: Sarvier; 1997. Pp. 519-34.

26. Fortes CBB, Samuel SMW. Avaliação de meios para armazenagem de resíduos de amálgama de prata. Rev Fac Odontol Porto Alegre. 1999;40(1):36-40.

27. Rink MCM, Borges VB, Figueira FC, Freitas W, Maciel LCN, Mainieri MP, et al. Contaminação pelo mercúrio: atitudes e cuidados entre cirurgiões dentistas. Rev Centro Cienc Biomed Univ Fed Uberlândia. 1994;10(1): 29-34.

28. Organização Pan-Americana da Saúde, Organização Mundial da Saúde. Guia para o manejo interno de resíduos sólidos em estabelecimentos de saúde. Brasília: OPAS; 1997.

29. Miller $\mathrm{CH}$, Palenik CJ. Control de la infección y manejo de materiales peligrosos para el equipo de profesionales de salud dental. $2^{\mathrm{a}}$ ed. Madrid: Harcourt; 2000.

Manuscrito recebido em 3 de julho de 2004. Aceito em versão revisada em 7 de dezembro de 2004
ABSTRACT

\section{Dental waste management in municipal health clinics in Belo Horizonte, Brazil}

Keywords
Objective. To investigate whether municipal health clinics in the city of Belo Horizonte, Brazil, comply with the legal requirements for managing dental wastes.

Method. We collected information from 54 of the city's 105 municipal health clinics that provide dental care. At each clinic we interviewed the clinic manager, one dental assistant, and one general assistant. Based on the requirements outlined in the Belo Horizonte Health Waste Management Manual, we assessed characteristics in the following three areas: (1) technical and operational (waste classification and characterization, minimization, segregation, pretreatment, conditioning, collection and internal and external transportation, and external storage); (2) general and organizational (inspection, amount of clinic space, environmental permits, floor plan showing wastegenerating areas, and whether the clinic had a technical specialist responsible for managing the health wastes); and (3) human resources (employee vaccination records and oversight, occupational safety and occupational medicine program, environmental risk prevention program, medical oversight and occupational health program, hospital (clinic) infection control committee, and training in health waste management).

Results. The clinics produced an average of 270 liters of solid waste per day. None of the clinics surveyed had a plan for managing health wastes. The only requirements with which all the clinics complied were: segregation of needles and mercury, adequately identified cardboard containers used for disposal of cutting and piercing items, and daily internal collection and transportation of wastes.

Conclusions. When the risks associated with each class of waste have not been established, all the wastes should be considered potentially dangerous. Further, a law by itself does not guarantee that the public's health will be protected. Before public agencies impose legal requirements, it is necessary to know if the agencies themselves are capable of enforcing those requirements. Any proposed waste management legislation should be based on scientific research.

Waste management; legislation, medical; dental waste; Brazil. 